\title{
Statistics of the fractional polarization of extragalactic dusty sources in Planck HFI maps
}

\author{
L. Bonavera, ${ }^{1 \star}$ J. González-Nuevo, ${ }^{1}$ B. De Marco, ${ }^{2}$ F. Argüeso ${ }^{3}$ and L. Toffolatti ${ }^{1,4}$ \\ ${ }^{1}$ Departamento de Física, Universidad de Oviedo, C. Federico García Lorca 18, E-33007 Oviedo, Spain \\ ${ }^{2}$ Nicolaus Copernicus Astronomical Center, Polish Academy of Sciences, Bartycka 18, PL-00-716 Warsaw, Poland \\ ${ }^{3}$ Departamento de Matemáticas, Universidad de Oviedo, C. Federico García Lorca 18, E-33007 Oviedo, Spain \\ ${ }^{4}$ INAF/IASF Bologna, Via Gobetti 101, Bologna, Italy
}

Accepted 2017 August 10. Received 2017 August 7; in original form 2017 May 25

\begin{abstract}
We estimate the average fractional polarization at 143,217 and $353 \mathrm{GHz}$ of a sample of 4697 extragalactic dusty sources by applying stacking technique. The sample is selected from the second version of the Planck Catalogue of Compact Sources at $857 \mathrm{GHz}$, avoiding the region inside the Planck Galactic mask $\left(f_{\text {sky }} \sim 60\right.$ per cent). We recover values for the mean fractional polarization at 217 and $353 \mathrm{GHz}$ of $(3.10 \pm 0.75)$ per cent and $(3.65 \pm 0.66)$ per cent, respectively, whereas at $143 \mathrm{GHz}$ we give a tentative value of $(3.52 \pm 2.48)$ per cent. We discuss the possible origin of the measured polarization, comparing our new estimates with those previously obtained from a sample of radio sources. We test different distribution functions and we conclude that the fractional polarization of dusty sources is well described by a log-normal distribution, as determined in the radio band studies. For this distribution we estimate $\mu_{217 \mathrm{GHz}}=0.3 \pm 0.5$ [that would correspond to a median fractional polarization of $\Pi_{\text {med }}=(1.3 \pm 0.7)$ per cent $]$ and $\mu_{353 \mathrm{GHz}}=0.7 \pm 0.4\left(\Pi_{\text {med }}=(2.0 \pm 0.8)\right.$ per cent $)$, $\sigma_{217 \mathrm{GHz}}=1.3 \pm 0.2$ and $\sigma_{353 \mathrm{GHz}}=1.1 \pm 0.2$. With these values we estimate the source number counts in polarization and the contribution given by these sources to the Cosmic Microwave Background B-mode angular power spectrum at 217, 353, 600 and $800 \mathrm{GHz}$. We conclude that extragalactic dusty sources might be an important contaminant for the primordial B-mode at frequencies $>217 \mathrm{GHz}$.
\end{abstract}

Key words: polarization-infrared: galaxies.

\section{INTRODUCTION}

The polarization properties of extragalactic dusty sources, i.e. sources dominated by thermal dust emission, at high frequencies $(>100 \mathrm{GHz})$ are still very poorly constrained (if not at all) by observations. For M82 a value for the fractional polarization, $\Pi$, of 0.4 per cent at $850 \mu \mathrm{m}$ has been measured by Greaves \& Holland (2002) with the polarimeter for the Submillimeter Common-User Array (SCUBA). The polarization data from Planck provide the first all-sky map of the polarized dust emission of our Galaxy (Planck Collaboration XIX 2015). From the Planck dust polarization maps De Zotti et al. (2016) has recently inferred an estimate of the fractional polarization of our Galaxy: they found an average polarization degree of 2.7 per cent for the Stokes Q parameter by integrating over a spatial band centred in the Galactic plane and with $20^{\circ}$ of width in latitude. This translates into a mean fractional polarization, $\langle\Pi\rangle$, of 1.4 per cent if all galaxies were polarized

^E-mail: laurabonavera@gmail.com like the Milky Way, by considering all the possible orientations of galactic discs with respect to the sky sphere.

The intrinsic Cosmic Microwave Background (CMB) polarized signal is very weak and primordial B-modes are yet to be discovered. The current upper limit on the tensor-to-scalar ratio from the second generation of the Background Imaging of Cosmic Extragalactic Polarization (BICEP2) and Keck Array experiments is $r<0.07$ (BICEP2/Keck Collaboration et al. 2015; BICEP2 Collaboration et al. 2016). Moreover, current measurements of the Galactic foreground emission (Choi \& Page 2015; Planck Collaboration XXX 2016) imply that primordial B-modes would be sub-dominant with respect to foregrounds on all angular scales and over all observational frequencies in the microwave regime. For this reason, the detection of B-modes should be regarded as a component separation problem (see Remazeilles et al. 2017, and references therein). Therefore, future CMB all-sky surveys in polarization (e.g. the proposed European Space Agency, European Space Agency, ESA, Cosmic ORigin Explorer, CORE, mission; see André, Baccigalupi \& Banday 2014) - with the capability to reach tensor-to-scalar ratios down to $r \sim 0.01$ - will need a much better characterization of the contaminating signal due 
to the (dominant) Galactic diffuse emission but also to compact extragalactic sources (see De Zotti et al. 2015, 2016, for comprehensive reviews on this subject).

In general, the contamination due to compact source to the polarization signal of the CMB does not affect large angular scales (near the reionization peak), but can play an important role at the intermediate and small angular scales of the lensing-induced B-mode signal (Curto et al. 2013). Indeed, dusty galaxies are expected to be the dominant foreground for $r \sim 0.001$ at small angular scales ( $l>47$ Curto et al. 2013; Remazeilles et al. 2017), once delensing has been applied to the data.

However, the polarization properties of populations of faint compact sources at mm wavelengths are poorly constrained. The currently available all-sky catalogues of compact sources at $\mathrm{mm} / \mathrm{sub}$ mm wavelengths are still limited to the shallow surveys provided by the Wilkinson Microwave Anisotropy Probe (WMAP; Bennett et al. 2003) and the ESA Planck (Planck Collaboration I 2016) missions. In the second, updated, version of the Planck Catalogue of Compact Sources (PCCS2; Planck Collaboration XXVI 2016) the number of detected compact sources in polarization is very low (i.e. 25 at $143 \mathrm{GHz}, 11$ at $217 \mathrm{GHz}$ and just one at $353 \mathrm{GHz}$ at the 99.99 per cent confidence level) and, thus, their polarization properties are poorly characterized. Moreover, it is obvious that only compact - either Galactic or extragalactic - sources with a high $\Pi$ can be detected and, thus, the statistical characterization of the underlying population will be biased towards these highly polarized objects.

In order to gather information about the statistical properties of the fractional polarization of compact sources in the microwave band, it is useful to exploit the full information embedded in CMB all-sky polarization maps by applying stacking techniques, i.e. by co-adding the signal from many weak or undetected objects to obtain a statistical detection. Recently, Bonavera et al. (2017, hereafter B17) have applied stacking to Planck maps to recover $\langle\Pi\rangle$ from 30 to $353 \mathrm{GHz}$ of a primary sample of 1560 compact radio sources. The sources were selected in the PCCS2 at $30 \mathrm{GHz}$ and were divided according to their location, i.e. whether located outside or inside the Galactic region of the sky defined by the Planck GAL60 mask and the region around the Magellanic Clouds. They found that $\langle\Pi\rangle$ is approximately constant with frequency in both samples, with a weighted mean value for all the Planck channels of 3.08 per cent outside and 3.54 per cent inside the Galactic mask, respectively. In the extragalactic region they estimated the parameters ( $\mu$ and $\sigma$ ) for the log-normal distribution of $\Pi$, finding a weighted mean value of 1.0 for $\sigma$ and 0.7 for $\mu$, that would imply a weighted median value for $\Pi$ of 1.9 per cent.

On one hand, these kinds of sources are yet poorly studied due to the lack of polarized data, especially considering that Planck is the only instrument with all-sky observation above $217 \mathrm{GHz}$ in polarization. On the other hand, their characterization would be very important not only per sè, to investigate their properties and their polarization emission mechanisms, but also as contaminant to the CMB for the recovery of the B-mode signal. Therefore, in order to improve the knowledge in polarization of such population of sources, with this work we extend the stacking approach to a sample of dusty galaxies, with the goal of estimating their fractional polarization, source number counts and their contribution to the primordial CMB polarized signal. The outline of the paper is as follows: in Section 2, we discuss the methods adopted for selecting the sky patches, for defining the sub-samples we are going to analyse and for determining their mean fractional polarization; in Section 3, we present our results and in Section 4 our conclusions.

\section{METHODS}

\subsection{Data}

Our sample is based on the PCCS2 (Planck Collaboration XXVI 2016) catalogue ${ }^{1}$ at $857 \mathrm{GHz}$ that contains 4891 sources with a flux density at the 90 per cent completeness level of $791 \mathrm{mJy}$ in total intensity. In order to avoid radio sources in our sample, we exclude those sources that have a counterpart in the $30 \mathrm{GHz}$ PCCS2 catalogue with a 33 arcmin search radius (151 sources). Of these 4740 sources, for consistency with what was done in the radio bands by B17, we consider for our analysis only those sources lying in the region outside the Planck Galactic mask that leaves the 60 per cent of the sky unmasked and we also exclude the $5 \mathrm{deg}$ radius region around the position of the Large Magellanic Cloud and the $3 \mathrm{deg}$ radius region around the Small Magellanic Cloud.

To check for further possible radio sources among these 4706 sources, we search in the PCCS2 $217 \mathrm{GHz}$ catalogue for sources in the PCCS2 $143 \mathrm{GHz}$ within a 7.1 arcmin search radius from the centre of the sources at $217 \mathrm{GHz}$. The resulting 944 sources are then cross-matched with our sample (the one selected at $857 \mathrm{GHz}$ and without the sources in the $30 \mathrm{GHz}$ channel). We find only 18 sources in common. Of these, only 9 have a radio spectrum $(\alpha<1)$, but their flux densities are too faint (the maximum flux density is lower than $400 \mathrm{mJy}$ ) to be detected at $857 \mathrm{GHz}$. In any case, we remove them to minimize any potential contamination when performing stacking at the lower frequencies. Our final sample consists of 4697 sources.

\subsection{Stacking}

In this work we apply the same methodology discussed in B17: we use stacking (see Dole et al. 2006; Marsden et al. 2009; Béthermin et al. 2012, and references therein) to reduce the noise/background, since it is expected to fluctuate around the mean with positive and negative values, and enhance the signal we want to study, correspondingly.

In our case we want to perform statistical estimates of polarization with Planck. It should be noticed that our target sources are all detected by Planck in total intensity at $857 \mathrm{GHz}$, but they are not necessarily detected in the lower frequency channels. At 857 and $545 \mathrm{GHz}$ Planck instruments are not sensitive to polarization. For this reason we compute $\langle\Pi\rangle$ of our source population at 143, 217 and $353 \mathrm{GHz}$. Due to the rising spectra with frequency of dusty sources, we do not expect to have a measurable signal either in total intensity or in total polarization at frequencies lower than $143 \mathrm{GHz}$.

To be consistent with B17, we perform stacking selecting the same small patch of $63 \times 63$ pixels (with a pixel size of 1.72 arcmin) around each source position. We then add up all the patches to obtain the total flux density. To reduce the instrumental noise (a second order effect) we convolve the resulting patch with a Normal filter whose $\sigma_{\text {filter }}$ is given by $\sigma_{\text {beam }} / 2$, where $\sigma_{\text {beam }}$ is $F W H M_{\text {instrument }} / 2 \sqrt{2 \ln 2}$. The $F W H M_{\text {instrument }}$ is $7.30,5.02$ and 4.94 arcmin at 143, 217 and $353 \mathrm{GHz}$, respectively (Planck Collaboration I 2016).

Following B17, we remove plausible contaminants to our stacked measurements that result in a strong background in the final stacked image. We subtract the mean of the background computed in the

\footnotetext{
${ }^{1}$ Based on observations obtained with Planck (http://www.esa.int/Planck), an ESA science mission with instruments and contributions directly funded by ESA Member States, NASA and Canada. Available at http://pla.esac.esa.int/pla/\#home (Planck Legacy Archive).
} 
external region of the final patch ( $3 \sigma_{\text {beam }}$ away from the patch centre) from the total flux density. From these residual maps we then compute the total flux densities in total intensity $S$ and polarization $P_{0}$ as the maximum values at the patch centre. We then obtain the averaged values $\left\langle P_{0}\right\rangle$ and $\langle S\rangle$ over our sample.

Finally, we compute $\langle\Pi\rangle=\left\langle P_{0}\right\rangle /\langle S\rangle$. Its error is given by $\sqrt{\left(\left\langle P_{0}\right\rangle /\langle S\rangle\right)^{2} \cdot\left(\sigma_{P_{0}}^{2} /\left\langle P_{0}\right\rangle^{2}+\sigma_{S}^{2} /\langle S\rangle^{2}\right)}$, where $\sigma_{P_{0}}$ and $\sigma_{S}$ are the standard deviations for total intensity and polarization computed in the external region of the stacked patches. We also compute the quantity $\sqrt{\left\langle\Pi^{2}\right\rangle}=\sqrt{\left\langle P_{0}^{2}\right\rangle /\left\langle S^{2}\right\rangle}$ by applying the same methodology. It can be a useful quantity to define the parameters for the fractional polarization distribution. Its error is given by $\sqrt{\frac{1}{4\left\langle P_{0}^{2}\right\rangle\left\langle S^{2}\right\rangle}\left(\sigma_{P_{0}^{2}}^{2}+\left\langle P_{0}^{2}\right\rangle^{2} /\left\langle S^{2}\right\rangle^{2} \sigma_{S^{2}}^{2}\right)}$.

Please note that most of our sources are not directly detectable and therefore we cannot estimate directly $\langle\Pi\rangle=\left\langle P_{0} / S\right\rangle$ and $\left\langle\Pi^{2}\right\rangle=$ $\left\langle P_{0}^{2} / S^{2}\right\rangle$. For this reason, in our stacking procedure we decided to calculate $\langle\Pi\rangle=\left\langle P_{0}\right\rangle /\langle S\rangle$ and $\left\langle\Pi^{2}\right\rangle=\left\langle P_{0}^{2}\right\rangle /\left\langle S^{2}\right\rangle$ that are good approximations for $\langle\Pi\rangle=\left\langle P_{0} / S\right\rangle$ and $\left\langle\Pi^{2}\right\rangle=\left\langle P_{0}^{2} / S^{2}\right\rangle$, assuming that $\Pi$ and $S$ could be considered independent variables as for the case in the radio band (B17). Besides, the residual errors introduced by these assumptions are much smaller than the noise bias (see B17 and Section 2.3 for more details) and the bias subtraction methodology described in Section 2.3 also corrects any residual deviation from the theoretical value.

\subsection{Source injection}

As described in detail in B17, the construction of $P=\sqrt{Q^{2}+U^{2}}$ introduces a bias usually called noise bias which has to be taken into account in order to obtain correct measurements. To estimate this bias, we carry out simulations with injected sources. This allows us to correct for any inaccuracy introduced by our assumptions, as stated above. We injected simulated compact sources in our real maps, at random positions but avoiding the positions of real sources.

The sources in our sample are those detected by Planck at $857 \mathrm{GHz}$ and they are mainly dusty local galaxies. They are simulated in total intensity at each frequency independently following Negrello et al. (2013). The latter uses the Planck Early release Compact Source Catalogue (ERCSC; Planck Collaboration VII 2011) to carefully determine the luminosity function, and therefore the source number counts, of local dusty galaxies at 857, 545 and $353 \mathrm{GHz}$.

The flux densities in polarization are first simulated as in B17 following a log-normal distribution for different values of $\langle\Pi\rangle$ in each simulation. In order to estimate the noise-bias correction, we inject simulated sources in the real maps by assuming a uniform random polarization angle. We compute $\mathrm{Q}$ and $\mathrm{U}$ for each source creating a simulated sources map starting with the simulated catalogue and convolve it with the FWHM of the instrument (different for each $v$ ) and add it to the real Q and U maps. For each simulation we randomly vary both the location $(\mu)$ and the scale $(\sigma)$ parameters of the log-normal distribution. We then apply the same methodology described in Section 2.2 to obtain a recovered (biased) value. We repeat this procedure for at least 100 simulations at each frequency. The single theoretical simulated values, $\langle\Pi\rangle=\left\langle P_{s} / S_{s}\right\rangle$, and the recovered ones are shown as grey points. The blue points are simply the binned values, equally spaced in the $y$-axis.

By comparing the theoretical simulated $\langle\Pi\rangle=\left\langle P_{s} / S_{s}\right\rangle$ with the recovered values we are able to obtain the noise bias correction relationships shown as blue points in Fig. 1. Finally, we estimate our debiased measurements (red points) using a linear interpolation of the derived noise bias correction relationships.

Then, we also check how the results change when using a different distribution for the fractional polarization to correct the biased values. We chose to study the cases for a Normal and a gamma distribution. We adopt the same procedure as for the log-normal distribution, randomly vary the $\mu$ parameter for the Normal distribution (and assuming $\sigma=0.5$ ) and the shape, $k$, and scale, $\theta$, parameters for the gamma distributions. We perform at least 50 simulations for each case.

We also compute the correction to $\sqrt{\left\langle\Pi^{2}\right\rangle}$ in the same way as for $\langle\Pi\rangle$.

As in B17 from the recovered values of $\langle\Pi\rangle$ and $\sqrt{\left\langle\Pi^{2}\right\rangle}$ we can compute the parameters $\mu$ and $\sigma$ characterizing the log-normal distribution:

$\mu=\ln \left(\frac{\langle\Pi\rangle^{2}}{\sqrt{\left\langle\Pi^{2}\right\rangle}}\right)$

$\sigma=\sqrt{\ln \left(\frac{\left\langle\Pi^{2}\right\rangle}{\langle\Pi\rangle^{2}}\right)}$

and their errors are, respectively

$\operatorname{var}(\mu)=\frac{4}{\langle\Pi\rangle^{2}} \operatorname{var}(\langle\Pi\rangle)+\frac{1}{\left\langle\Pi^{2}\right\rangle} \operatorname{var}\left(\sqrt{\left\langle\Pi^{2}\right\rangle}\right)$

$\operatorname{var}(\sigma)=\frac{1}{\sigma^{2}}\left(\frac{\operatorname{var}(\langle\Pi\rangle)}{\langle\Pi\rangle^{2}}+\frac{1}{\left\langle\Pi^{2}\right\rangle} \operatorname{var}\left(\sqrt{\left\langle\Pi^{2}\right\rangle}\right)\right)$.

From $\mu$ we can then compute the median fractional polarization as

$\Pi_{m}=\exp (\mu)$

Note that for the Planck High Frequency Instrument channels the leakage effect is negligible when estimating the polarized flux density of compact sources (see Planck Collaboration XXVI 2016), so there is no need to take it into account in the simulations.

\section{RESULTS}

Our sample consists of 4697 sources as described in 2.1. We perform stacking in the Planck channels from 143 to $353 \mathrm{GHz}$, both in total intensity and polarization. From the patches resulting from stacking we estimate $\langle S\rangle,\left\langle P_{0}\right\rangle,\langle\Pi\rangle$ and $\left\langle S^{2}\right\rangle,\left\langle P_{0}^{2}\right\rangle, \sqrt{\left\langle\Pi^{2}\right\rangle}$ and we compute the errors from the standard deviation of the residual background fluctuations, as described in Section 2.2. The results are summarized in the left part of Table 1.

\section{1 $\langle\Pi\rangle$ and $\left\langle\Pi^{2}\right\rangle$ estimate}

To estimate $\langle\Pi\rangle$ from the bias-uncorrected values we use the procedure described in Section 2.3, whose results are shown in Fig. 1 and listed in Table 1. The same approach has been applied to correct also the values obtained for $\sqrt{\left\langle\Pi^{2}\right\rangle}$ (see Table 1 and Fig. 2).

Due to the large errors the mean fractional polarization at $143 \mathrm{GHz}$ is not well constrained $[\langle\Pi\rangle=(3.52 \pm 2.48)$ per cent and $\sqrt{\left\langle\Pi^{2}\right\rangle}=(7.31 \pm 3.89)$ per cent $]$.

Fig. 3 is a summary of the noise bias corrected results on $\langle\Pi\rangle$ (top panel) and $\sqrt{\left\langle\Pi^{2}\right\rangle}$ (bottom panel) we obtain with stacking on the current sample, compared with those obtained by B17. We obtain 


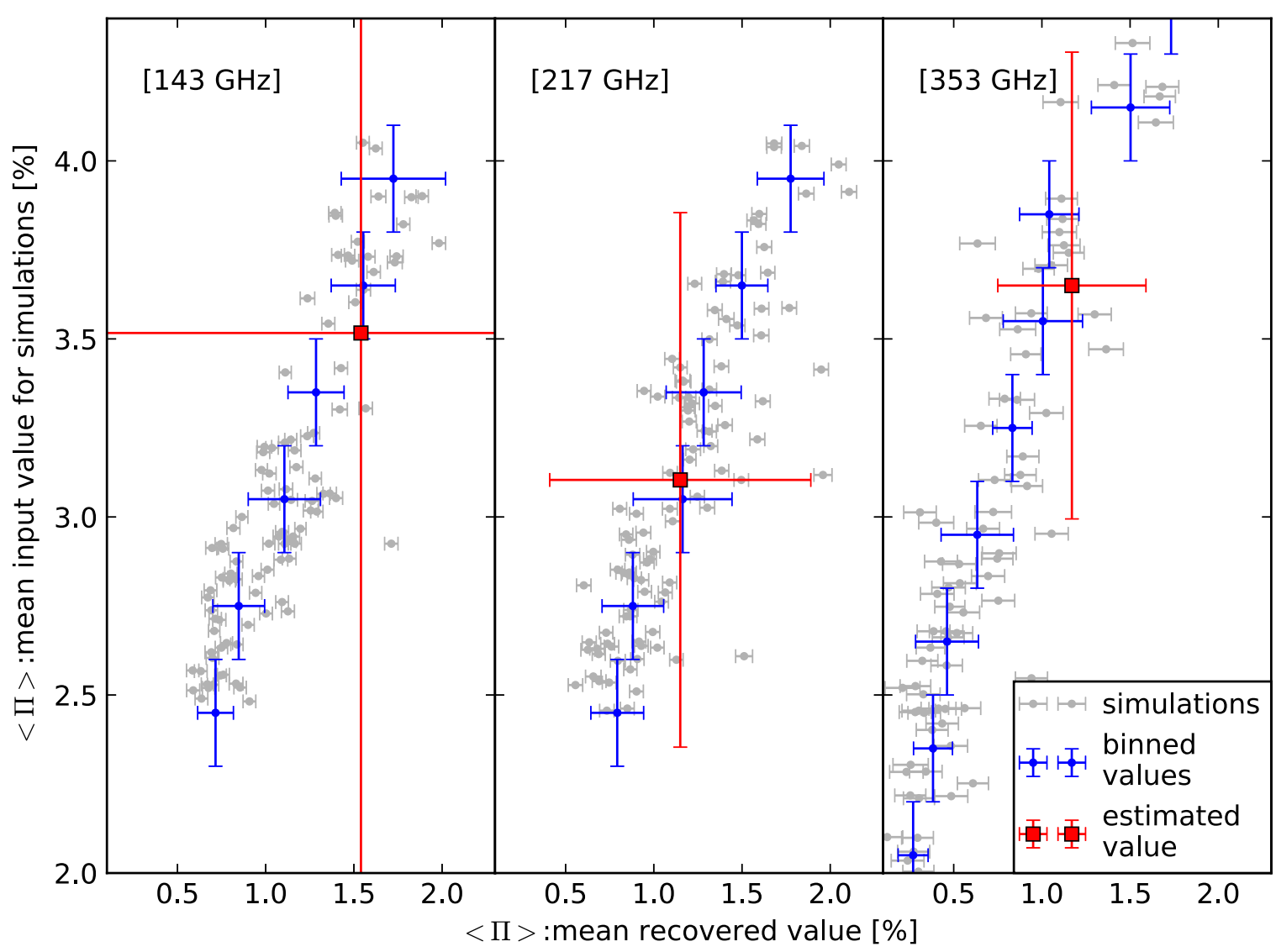

Figure 1. Results obtained outside the adopted mask (extragalactic region of the sky) from 143 to $353 \mathrm{GHz}$. The grey points are obtained with each individual simulation (using a log-normal distribution for the mean fractional polarization): on the $y$-axis we plot the mean input $\langle\Pi\rangle$ value for simulations and the $x$-axis is the value recovered with stacking for different values of $\mu$ and $\sigma$, as described in the text. For all the panels, the linear interpolation of these points gives us the correction for the noise bias that has to be applied to the observed values (red squares). The blue points are obtained by averaging over the simulations points with a binning step of 0.3 in the $y$-axis.

Table 1. From left to right: frequency, mean fractional polarization with rms errors uncorrected and corrected for the noise bias, square root of the mean quadratic fractional polarization with $1 \sigma$ errors uncorrected and corrected for the noise bias, $\mu$ and $\sigma$ parameters of the log-normal function characterizing the mean fractional polarization distributions (see the text) and their $1 \sigma$ errors and median fractional polarization computed as $\Pi_{m}$ and its error. The results are for the case outside the Planck Galactic mask with $f_{\text {sky }}=60$ per cent and excluding the regions around the Magellanic Clouds as described in the text.

\begin{tabular}{|c|c|c|c|c|c|c|c|c|c|c|c|c|c|c|}
\hline \multirow[b]{3}{*}{$\begin{array}{l}\text { Frequency } \\
(\mathrm{GHz})\end{array}$} & \multirow{2}{*}{\multicolumn{2}{|c|}{ Uncorrected }} & \multirow{2}{*}{\multicolumn{6}{|c|}{$\begin{array}{l}\text { Extragalactic region } \\
\text { Uncorrected }\end{array}$}} & \multirow{2}{*}{\multicolumn{6}{|c|}{ Log-normal parameters }} \\
\hline & & & & & & & & & & & & & & \\
\hline & $\begin{array}{c}\langle\Pi\rangle \\
\text { percent }\end{array}$ & $\begin{array}{c}\text { Error } \\
\text { percent }\end{array}$ & $\begin{array}{c}\langle\Pi\rangle \\
\text { percent }\end{array}$ & $\begin{array}{c}\text { Error } \\
\text { percent }\end{array}$ & $\begin{array}{l}\sqrt{\left\langle\Pi^{2}\right\rangle} \\
\text { percent }\end{array}$ & $\begin{array}{c}\text { Error } \\
\text { percent }\end{array}$ & $\begin{array}{l}\sqrt{\left\langle\Pi^{2}\right\rangle} \\
\text { percent }\end{array}$ & $\begin{array}{c}\text { Error } \\
\text { percent }\end{array}$ & $\mu$ & Error & $\sigma$ & Error & $\begin{array}{c}\Pi_{m} \\
\text { percent }\end{array}$ & $\begin{array}{l}\text { Error } \\
\text { percent }\end{array}$ \\
\hline 143 & 1.54 & 2.42 & 3.52 & 2.48 & 12.56 & 12.14 & 7.31 & 3.89 & 0.53 & 1.51 & 1.21 & 0.73 & 1.6 & 2.5 \\
\hline 217 & 1.15 & 0.74 & 3.10 & 0.75 & 7.79 & 2.42 & 7.38 & 1.32 & 0.26 & 0.52 & 1.32 & 0.23 & 1.3 & 0.7 \\
\hline 353 & 1.17 & 0.42 & 3.65 & 0.66 & 5.44 & 1.28 & 6.87 & 1.35 & 0.66 & 0.41 & 1.12 & 0.24 & 2.0 & 0.8 \\
\hline
\end{tabular}

$\langle\Pi\rangle$ of $(3.10 \pm 0.75)$ per cent and $(3.65 \pm 0.66)$ per cent at 217 and $353 \mathrm{GHz}$, respectively.

Interestingly, very similar values were obtained from the analysis of the radio sample in B17, i.e. (3.07 \pm 0.29$)$ per cent for $217 \mathrm{GHz}$ and $(3.52 \pm 1.20)$ per cent for $353 \mathrm{GHz}$. We cannot extract any value of $\Pi$ from the PCCS2 catalogue for the dusty sources: we verified that all the sources in the PCCS2 catalogue at 143, 217 and $353 \mathrm{GHz}$ with reliable detection in polarization have a counterpart at $30 \mathrm{GHz}$, therefore we can consider them radio sources.

The similar results inferred from the analysis of the dusty and radio samples might imply a common origin for the observed polarization. In this scenario, polarization might be mainly caused by the synchrotron emission from the bursts of star formation and/or nuclear activity of dusty galaxies, with negligible contribution from dust. However, dust thermal emission contributes significantly to the total intensity at these frequencies, decreasing the mean fractional polarization potentially produced by the synchrotron emission.

In fact, following Mancuso et al. (2017), we estimate the contribution from the synchrotron emission produced by the star formation with a moderate rate (SFR), which is an upper limit for local dusty galaxies (the kind of sources in our sample, Negrello et al. 2013). For SFR between 1 to $10 M_{\odot} \mathrm{yr}^{-1}$ and $z$ $\lesssim 0.1$ we obtain a 5 per cent of synchrotron emission in total intensity at $353 \mathrm{GHz}\left(I_{\mathrm{sync}}=0.05 I_{T}\right)$. If we assume that the polarization 


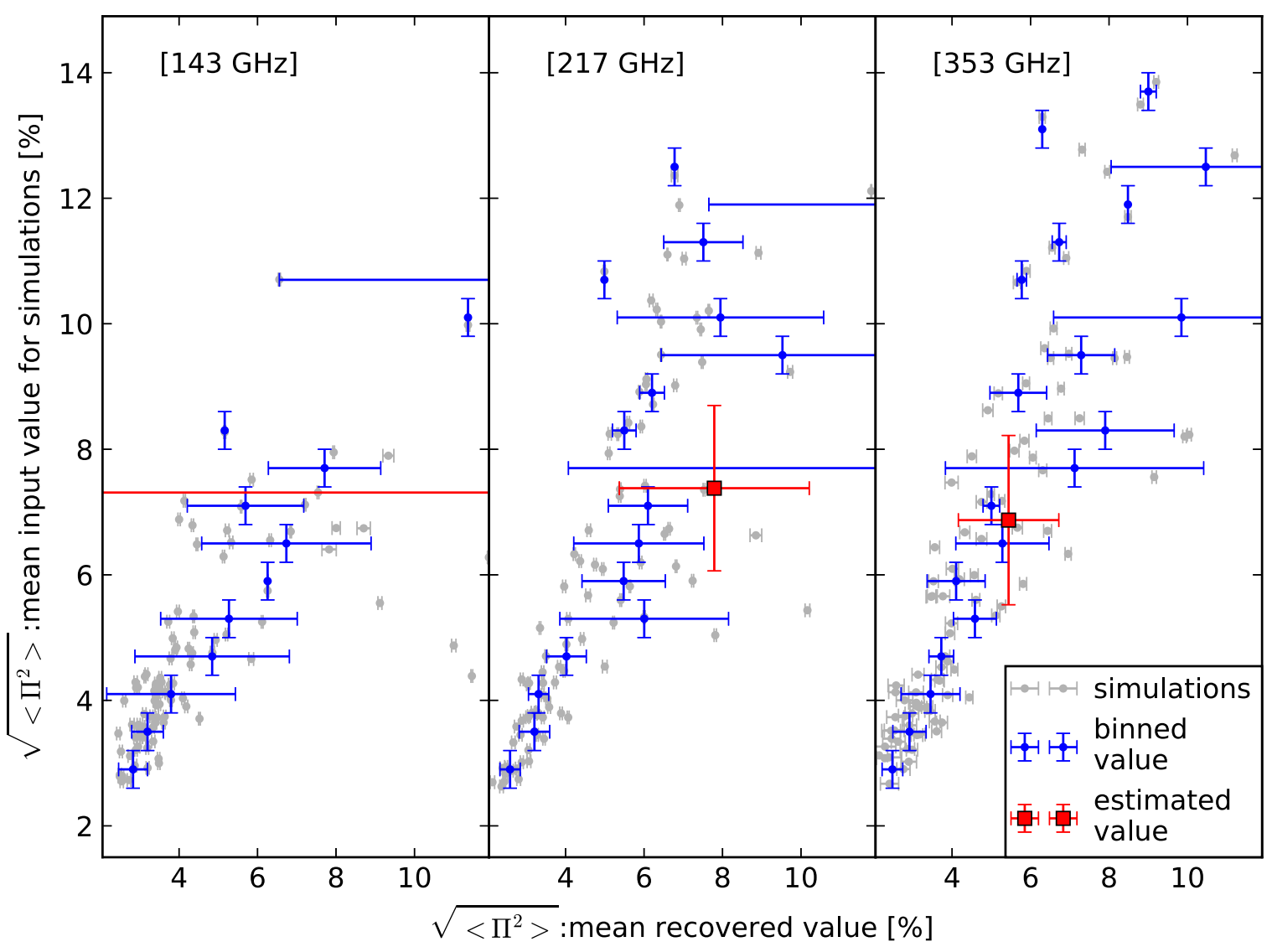

Figure 2. Results obtained for $\sqrt{\left\langle\Pi^{2}\right\rangle}$ outside the adopted mask (extragalactic region of the sky) from 143 to $353 \mathrm{GHz}$. The grey points are obtained with each individual simulation (using a log-normal distribution for the mean fractional polarization): the $y$-axis is the mean input $\sqrt{\left\langle\Pi^{2}\right\rangle}$ for simulations and the $x$-axis is the value recovered with stacking for different values of $\mu$ and $\sigma$, as described in the text. The linear interpolation of these points gives us the correction for the noise bias that has to be applied to the observed values (red squares). The blue points are obtained by averaging over the simulations points with a binning step of 0.3 in the $y$-axis.

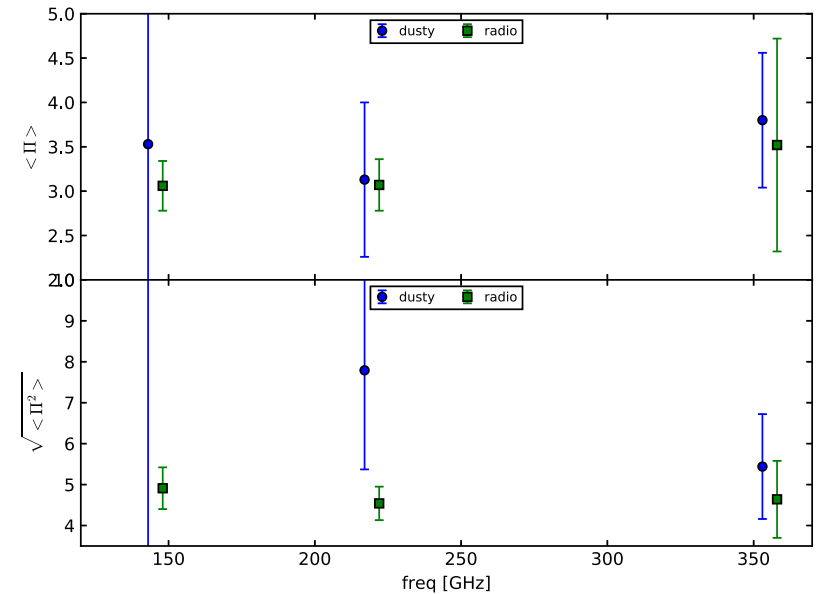

Figure 3. $\langle\Pi\rangle$ (top) and $\sqrt{\left\langle\Pi^{2}\right\rangle}$ (bottom) for each frequency outside the Galactic mask with $f_{\text {sky }}=60$ per cent and the Magellanic Clouds. The blue circles are obtained for the current sample, the green squares refer to the sample discussed in B17. emission is all due to synchrotron emission $\left(P_{\text {sync }}=P_{T}=\Pi\right.$. $\left.I_{T}=0.03 I_{T}\right)$, we find a $\Pi_{\text {sync }}=P_{\text {sync }} / I_{\text {sync }}=0.6$. Therefore, in order to recover the observed $\sim 3$ per cent mean fractional polarization at $353 \mathrm{GHz}$, the contribution from polarized synchrotron emission should be infeasibly high ( 60 per cent). The same calculation at $217 \mathrm{GHz}$ gives $I_{\text {sync }}=0.2 I_{T}$, implying a value for $\Pi_{\text {sync }}$ of 15 per cent. Since the mechanism responsible for the polarization emission at both frequencies for the same population of sources should be the same, this implies that the value we find at $353 \mathrm{GHz}$ rules out also the lower value for $217 \mathrm{GHz}$. This argument implies that dust contributes significantly to the observed polarization in the dusty sample.

Above $\sim 100 \mathrm{GHz}$ not only dusty galaxies, but even AGN with weak radio emission are dominated by dust emission from the torus or the host galaxy, as in typical sources dominated by thermal emission. Therefore, the observed polarization is likely associated with scattering processes off the dust grains and dust magnetic fields. On the other hand, the radio sample analysed in B17 consists mainly of Blazar sources. The latter are dominated by the jet emission at all frequencies, thus the observed polarization is to be ascribed to partially ordered magnetic fields in the jet (Tucci \& Toffolatti 2012, and references therein). This is expected to be the case when the shocks in the jet compress an initial random field (with 


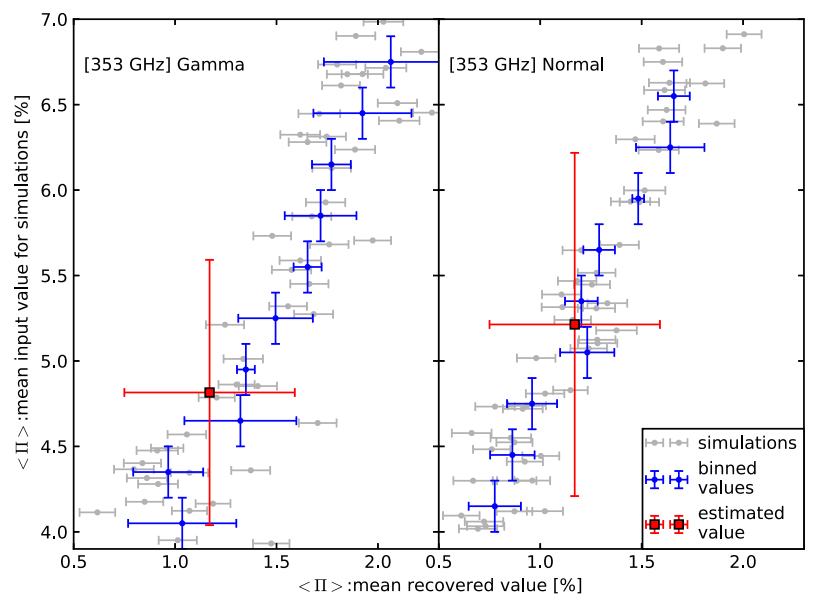

Figure 4. Results obtained outside the adopted mask (extragalactic region of the sky) for the $353 \mathrm{GHz}$ case. The grey points are obtained with each individual simulation: on the $y$-axis we plot the mean input $\langle\Pi\rangle$ value for simulations and the $x$-axis is the value recovered with stacking for different values of the mean for the Normal (left) and gamma (right) distributions. Linear interpolation and blue points as in Figs 1 and 2.

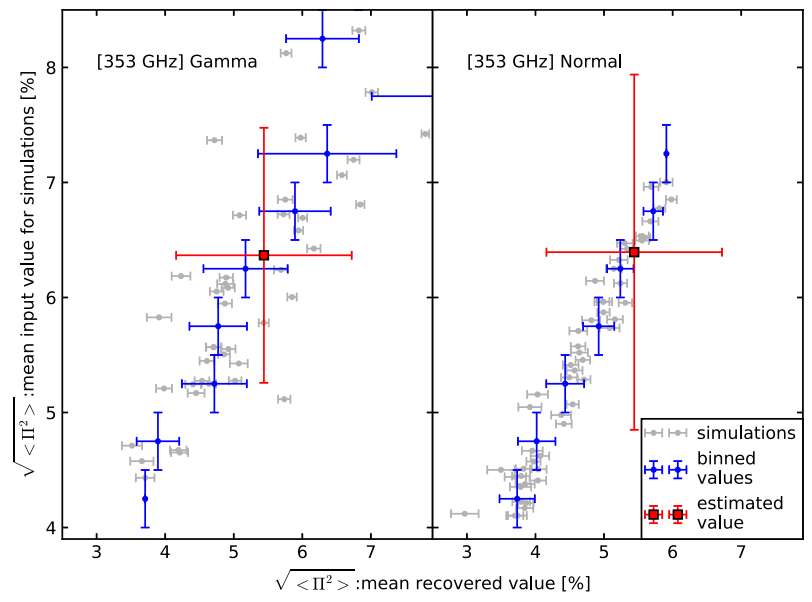

Figure 5. Results obtained outside the adopted mask (extragalactic region of the sky) for the $353 \mathrm{GHz}$ case. The grey points are obtained with each individual simulation: on the $y$-axis we plot the mean input $\sqrt{\left\langle\Pi^{2}\right\rangle}$ value for simulations and the $x$-axis is the value recovered with stacking for different values of the mean for the Normal (left) and gamma (right) distributions. Linear interpolation and blue points as in Figs 4 and 5.

$\mathrm{B}$ perpendicular to the jet axis) or sheared to lie in plane (with $\mathrm{B}$ parallel to the jet axis).

Since the physics that produces these polarization signals is completely different we can only conclude that this close similarity in the $\langle\Pi\rangle$ values can be just a coincidence.

As in B17, from the recovered values of $\langle\Pi\rangle$ and $\sqrt{\left\langle\Pi^{2}\right\rangle}$ we compute the parameters $\mu$ and $\sigma$ for the log-normal distribution and from $\mu$ we compute the median fractional polarization as $\Pi_{m}=\exp (\mu)$. The resulting values are summarized in the right part of Table 1 .

\subsection{Polarization distribution}

We also recover unbiased values for $\langle\Pi\rangle$ and $\sqrt{\left\langle\Pi^{2}\right\rangle}$ at $353 \mathrm{GHz}$ using two different distributions for the polarization fraction (see Figs 4 and 5). The aim is to test how other distributions work when estimating these quantities. We test the Normal and the gamma

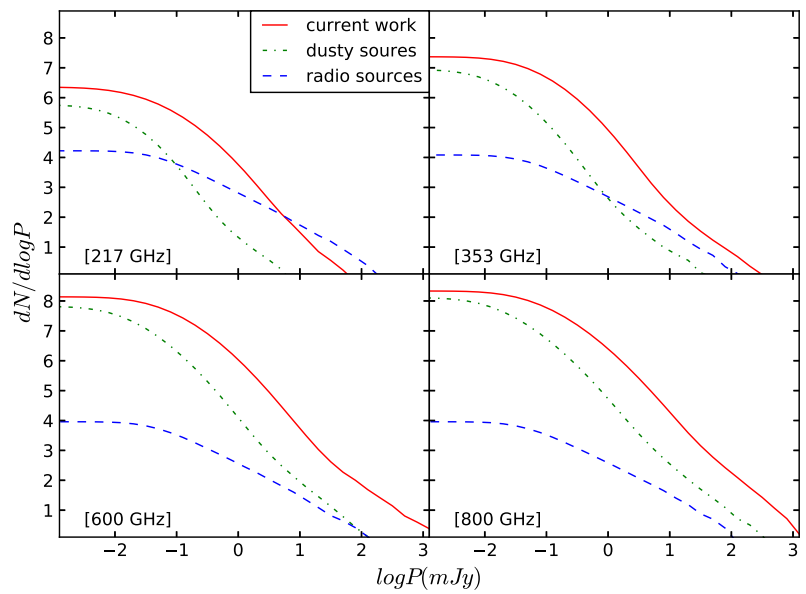

Figure 6. Source number counts estimated for our sample of sources (red solid line) compared with the CORE predictions (De Zotti et al. 2016) for the dusty (green dot-dashed line) and radio (blue dashed) sources. In the top panels we estimate the source number counts at 217 (left) and 353 (right) $\mathrm{GHz}$ with the results in Table 1. In the lower panel we show the estimation at 600 (left) and 800 (right) GHz obtained assuming the same values for the log-normal parameters as for the $353 \mathrm{GHz}$ case.

distributions. In the first case we obtain $(5.21 \pm 1.00)$ per cent for $\langle\Pi\rangle$ and $(6.39 \pm 1.54)$ per cent for $\sqrt{\left\langle\Pi^{2}\right\rangle}$. For the Normal case the following relations between the $E[x]=\langle\Pi\rangle$ and $E\left[x^{2}\right]=\left\langle\Pi^{2}\right\rangle$ hold: $E[x]=\mu$ and $E\left[x^{2}\right]=\mu^{2}+\sigma^{2}$. By substituting the values we obtain a negative value for $\sigma^{2}$, which is not possible and allow us to discard a Normal like distribution for the fractional polarization. The same argument can be applied to the gamma distribution, for which we find $(4.82 \pm 0.78)$ per cent for $\langle\Pi\rangle$ and $(6.37 \pm 1.11)$ per cent for $\sqrt{\left\langle\Pi^{2}\right\rangle}$. In this case the relations are $E[x]=k \theta$ and $E\left[x^{2}\right]=k \theta^{2}+k^{2} \theta^{2}$, that give a negative value for the product $k \theta^{2}$ when substituting the values we find. Considering that for the gamma family of distributions it should be $k>0$, we can confidently discard also this broad family of distributions for the fractional polarization.

\subsection{Source counts and CMB power spectrum contribution}

To estimate the source numbers counts in polarization, we proceed with simulations. At 217 and $353 \mathrm{GHz}$, we first simulate sources in total intensity according to the source number counts by Negrello et al. (2013). Then we simulate the fractional polarization assuming a log-normal distribution with the parameters listed in Table 1. Finally, we randomly associate the simulated $\Pi$ value with the flux density in total intensity of the simulated sources. We repeat this process 10 times and we estimate the source number counts in polarization by averaging over these 10 sets of simulations.

We also estimate the source number counts in polarization for 600 and $800 \mathrm{GHz}$ (CORE frequencies) assuming the $353 \mathrm{GHz}$ parameters for the log-normal distribution. The results are shown in Fig. 6: the red solid line are the source counts for our sample and they are compared with the dusty (green dot-dashed line) and radio (blue dashed line) sources predicted by De Zotti et al. (2016).

From the source number counts in polarization we estimate the contribution in polarization to the CMB power spectrum, following De Zotti et al. (1996):

$C_{l}^{P}=g^{2} \int_{0}^{P_{c}} P^{2} \frac{\mathrm{d} N}{\operatorname{dlog} P} \mathrm{~d} \log P$ 
Table 2. From left to right: frequency, $C_{l}$ contribution to the CMB power spectrum of our sample of dusty and of radio sources estimated from the CORE predictions (De Zotti et al. 2016).

\begin{tabular}{lcc}
\hline $\begin{array}{l}\text { Frequency } \\
(\mathrm{GHz})\end{array}$ & $\begin{array}{c}\text { Dusty sources } \\
\left(\mu \mathrm{K}^{2}\right)\end{array}$ & $\begin{array}{c}\text { Radio } \\
\left(\mu \mathrm{K}^{2}\right)\end{array}$ \\
\hline 217 & $5.57 \mathrm{e}-08$ & $4.38 \mathrm{e}-09$ \\
353 & $2.22 \mathrm{e}-06$ & $8.61 \mathrm{e}-09$ \\
600 & $2.06 \mathrm{e}-03$ & $5.66 \mathrm{e}-07$ \\
800 & $5.10 \mathrm{e}-01$ & $6.62 \mathrm{e}-05$ \\
\hline
\end{tabular}

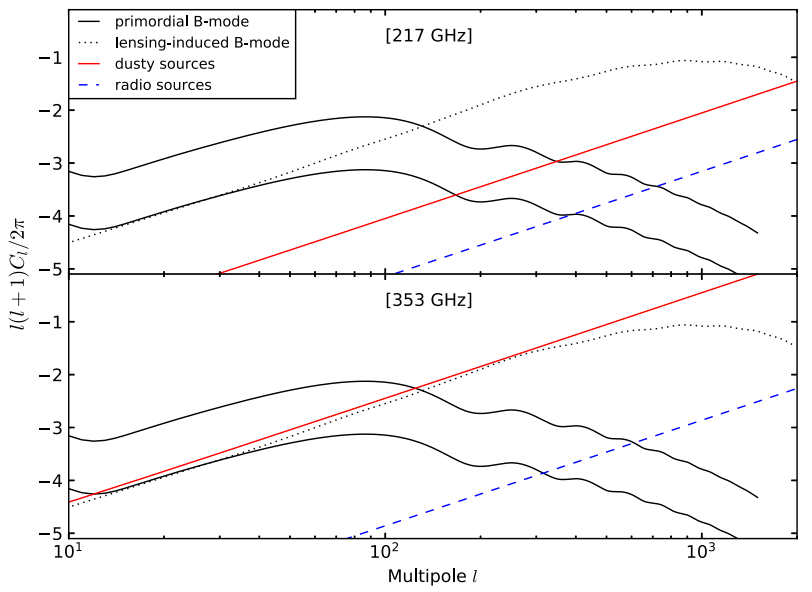

Figure 7. Power spectrum contribution from the dusty (red solid line) and radio (blue dashed line) sources to the power spectrum, compared with the primordial B-mode for $r=0.1,0.01$, from top to bottom (black solid line) and the lensing-induced B-mode (black dotted line).

where $g$ is the conversion factor from flux density to temperature units (Tegmark \& Efstathiou 1996) and $P_{c}=0.5 \mathrm{mJy}$ has been chosen to be similar to the CORE detection limit (De Zotti et al. 2016). The values we obtain for $217,353,600$ and $800 \mathrm{GHz}$ are listed in Table 2. In Fig. 7, we plot the $l(l+1) C_{l} / 2 \pi$ estimate for the dusty sources (red solid line) and for the radio sources in (De Zotti et al. 2016, blue dashed line) for the 217 (top panel) and 353 (bottom panel) $\mathrm{GHz}$ channels. In these two frequencies the contribution is comparable to the primordial B-mode for $r=0.1$ and $r=0.01$ (black solid lines) and the lensing-induced B-mode (black dotted line). At 217 the contribution from these kind of sources is negligible for the lensing-induced B-modes, but it becomes important for the primordial B-mode at $l \sim 350$ (right after the second peak) or $l \sim 150$ (just after the first peak) for $r=0.1$ and $r=0.01$, respectively.

At $353 \mathrm{GHz}$ the level of this contamination is the same as the one of lensing-induced B-modes. This means that it is even worse for the detection of the primordial B-mode, since the source contribution becomes important right after the first peak $(l \sim 100)$ already for the $r=0.1$ case. We omit to show results from 600 and $800 \mathrm{GHz}$, as at these frequencies the contribution to the power spectrum is much higher than the one from the B-mode, as expected.

\section{CONCLUSIONS}

The analysed sample of extragalactic dusty sources (selected from the $857 \mathrm{GHz}$ of the PCCS2 catalogue) shows polarization properties similar to those characterizing radio sources (Massardi et al. 2013; Galluzzi et al. 2017, B17). We measure polarization values of
$\langle\Pi\rangle=(3.10 \pm 0.75)$ per cent and $\langle\Pi\rangle=(3.65 \pm 0.66)$ per cent at 217 and $353 \mathrm{GHz}$ respectively, and of $\sqrt{\left\langle\Pi^{2}\right\rangle}=(7.38 \pm 1.32)$ per cent and $(6.87 \pm 1.35)$ per cent at 217 and $353 \mathrm{GHz}$, respectively. Moreover, as for the radio sources, the fractional polarization of extragalactic dusty sources follows a log-normal distribution. We find values for $\mu$ of $0.26 \pm 0.52$ and $0.66 \pm 0.41$ and for $\sigma$ of $1.32 \pm 0.23$ and $1.12 \pm 0.24$ at 217 and $353 \mathrm{GHz}$, respectively. However, radio and dusty sources are dominated by different components at these frequencies, i.e. by jet synchrotron and dust emission, respectively (see discussion in Section 3.1). Therefore we conclude that the inferred similarities of polarization properties are fortuitous.

We update with our new measurements the source number counts in polarization at 217 and $353 \mathrm{GHz}$ and compare them with the predictions for the CORE proposal (De Zotti et al. 2016). Moreover we make prediction for the level of the expected contamination to the B-mode angular power spectrum. We also extrapolate the results at higher frequencies $(600$ and $800 \mathrm{GHz})$. We find that at $217 \mathrm{GHz}$ the extragalactic dusty sources might be an important contaminant for the primordial B-mode, especially in the case of $r=0.01$ or lower. At $353 \mathrm{GHz}$ their contribution is at the level of the lensing-induced B-mode. As expected, their importance increases with frequency and at 600 and $800 \mathrm{GHz}$ their contribution to the angular power spectrum is much higher than the ones of the B-mode, both primordial and lensing-induced.

\section{ACKNOWLEDGEMENTS}

The authors acknowledge the referee R. Taylor for his useful comments.

LB, JGN, FA and LT acknowledge financial support from the I+D 2015 project AYA2015-65887-P (MINECO/FEDER). J.G.N also acknowledges financial support from the Spanish MINECO for a 'Ramon y Cajal' fellowship (RYC-2013-13256). BDM acknowledges support from the European Union's Horizon 2020 research and innovation programme under the Marie SkłodowskaCurie grant agreement No. 665778 via the Polish National Science Center grant Polonez UMO-2016/21/P/ST9/04025.

\section{REFERENCES}

André P., Baccigalupi C., Banday A., 2014, J. Cosmology Astropart. Phys., 2, 006

Bennett C. L. et al., 2003, ApJ, 583, 1

Béthermin M. et al., 2012, A\&A, 542, A58

BICEP2 Collaboration et al., 2016, ApJ, 833, 228

BICEP2/Keck Collaboration et al., 2015, Phys. Rev. Lett., 114, 101301

Bonavera L., González-Nuevo J., Argüeso F., Toffolatti L., 2017, MNRAS, 469, 2401 (B17)

Choi S. K., Page L. A., 2015, JCAP, 12, 020

Curto A., Tucci M., González-Nuevo J., Toffolatti L., Martínez-González E., Argüeso F., Lapi A., López-Caniego M., 2013, MNRAS, 432, 728

de Zotti G., Franceschini A., Toffolatti L., Mazzei P., Danese L., 1996, ApL\&C, 35, 289

De Zotti G. et al., 2015, JCAP, 6, 018

De Zotti G. et al., 2017, JCAP, preprint (arXiv:1609.07263)

Dole H. et al., 2006, A\&A, 451, 417

Galluzzi V. et al., 2017, MNRAS, 465, 4085

Greaves J. S., Holland W. S., 2002, Cecchini S., Cortiglioni S., Sault R., Sbarra C., eds, AIP Conf. Proc. Vol. 609, Astrophysical Polarized Backgrounds: Workshop on Astrophysical Polarized Backgrounds Am. Inst. Phys., New York, p. 267

Mancuso C. et al., 2017, ApJ, 842, 95

Marsden G. et al., 2009, ApJ, 707, 1729

Massardi M. et al., 2013, MNRAS, 436, 2915 
Negrello M. et al., 2013, MNRAS, 429, 1309

Planck Collaboration I, 2016, A\&A, 594, A1

Planck Collaboration VII, 2011, A\&A, 536, A7

Planck Collaboration XIX, 2015, A\&A, 576, A104

Planck Collaboration XXVI, 2016, A\&A, 594, A26

Planck Collaboration XXX, 2016, A\&A, 586, A133
Remazeilles M. et al., 2017, JCAP, preprint (arXiv:1704.04501)

Tegmark M., Efstathiou G., 1996, MNRAS, 281, 1297

Tucci M., Toffolatti L., 2012, Adv. Astron., 624987

This paper has been typeset from a $\mathrm{T}_{\mathrm{E}} \mathrm{X} / \mathrm{L} \mathrm{T} \mathrm{E} \mathrm{X}$ file prepared by the author. 